\title{
Low-energy nanoemulsification to design veterinary controlled drug delivery devices
}

\author{
This article was published in the following Dove Press journal: \\ International Journal of Nanomedicine \\ 14 October 2010 \\ Number of times this article has been viewed
}

\section{Thierry F Vandamme Nicolas Anton \\ University of Strasbourg, Faculty of Pharmacy, Illkirch Cedex, France; UMR CNRS 7I99, Laboratoire de Conception et Application de Molécules Bioactives, équipe de Pharmacie Biogalénique, Illkirch Cedex, France}

This work is selected as Controlled Release Society Outstanding Veterinary Paper Award 2010
Correspondence: Thierry F Vandamme University of Strasbourg, Faculty of Pharmacy, 74 Route du Rhin, BP 60024, F-6740I Illkirch Cedex, France

Tel +33368854106

Fax +33368854325

Email vandamme@unistra.fr
Abstract: The unique properties of nanomaterials related to structural stability and quantum-scale reactive properties open up a world of possibilities that could be exploited to design and to target drug delivery or create truly microscale biological sensors for veterinary applications. We developed cost-saving and solvent-free nanoemulsions. Formulated with a lowenergy method, these nanoemulsions can find application in the delivery of controlled amounts of drugs into the beverage of breeding animals (such as poultry, cattle, pigs) or be used for the controlled release of injectable poorly water-soluble drugs.

Keywords: nanoemulsion, nanomedicine, low-energy emulsification, veterinary, ketoprofen, sulfamethazine

\section{Introduction}

Comparative medicine has seen resurgence over the past 10 years largely because of the growth of evidence-based veterinary medicine and the gain in knowledge acquired from comparative genomics. Diseases such as diabetes, hypertension, skin atrophy, and cancer in some pet animals are similar to those in humans, which enables parallel development of therapeutic approaches. The synergy inherent to a single medicine could result in significant cost-savings for product development for both animals and humans.

A number of factors that must be taken into consideration when assessing what might emerge as new animal drug products in the future. These factors revolve around economic, societal, and technological issues. However, economic and societal aspects are decisive factors in business decisions, as without a market, new products will not be developed. Projection of transforming Transformational technologies such as analytical chemistry, computational sciences, molecular biology, genomics, and material engineering are key predictors of future developments. Economic, societal, and technological issues have led to pharmacology research for the treatment of conditions such as aggressive behavior, separation anxiety, or obsessive-compulsive disorder and the launch of dirlotapide, a drug targeted to reduce obesity in dogs, 4 years ago. The future trends for the pet animal market seem to be getting more sophisticated. Moreover, the pet animal market and animal health products have slim profit margins. Global competition for food is intense, reducing marginal return rates. In order to improve the quality of animal health, future developments will take into account the following:

1. Continued advances in pharmaceutical technology;

2. Nanotechnology;

3. General increased ability to control and target drug delivery;

4. Increased knowledge of pharmacogenomics. 
Nanotechnology, defined as manufacturing materials that are $<100 \mathrm{~nm}$ in one dimension and having unique physical properties due to this size, was first designed for human applications. At present, developing nanotechnologies for pets and farm animal markets seems to be a real opportunity for designing new products. These technologies could find applications in the development of solvent-free injectable dosage forms containing drugs of low water solubility and for the controlled delivery of drugs (eg, antibiotics, anthelmintics) into beverages of breeding animals (eg, farm animals poultry, cattle or pigs). The strategy commonly used in food industries for delivering such drugs is based on the use of premix, which is not optimized for the different animal species, or even for animals of different weights. The novelty of the present work lies in proposing a simple method that enables the exact amount of drug do be determined and delivered according the animal species and/or body weight.

In this context, the present study aimed to adapt a lowenergy nanoemulsion formulation method that we recently described $^{1,2}$ to such specifications. The nanoemulsion droplets are spontaneously obtained very easily by bringing into contact a liquid phase composed of a premix of drugs, lipids, and surfactants, with a second phase, which is either drinking water or the aqueous phase that has to be injected. Nanoemulsions are immediately generated, allowing the dispersion of the poorly water-soluble drinking drug model in a definitively stable way in the aqueous phase. In particular, one of the advantages of these systems is to control the amount of drug administered to the animals by changing the volume of the premix added to the drinking water. Obviously, these systems can be considered only if the premix can spontaneously form nanoemulsions without using external energy. For proof of concept, we used ketoprofen as a poorly water-soluble injectable drug model and sulfamethazine as poorly water-soluble drinking drug model. As discussed below, these two drug models were actually selected based on their physicochemical properties and their affinity for the lipophilic phases used for formulating the nanoemulsions.

\section{Materials and methods \\ Materials}

Nonionic surfactants were Cremophor ELP ${ }^{\circledR}$ (BASF, Ludwigshafen, Germany; polyoxyethylated-35 castor oil, with a specified hydrophilic-lipophilic balance [HLB] of 12-14) and Solutol HS $15^{\circledR}$ (BASF, Ludwigshafen, Germany; macrogol 15 hydroxystearate, HLB of 14-16), which were kindly provided by Laserson (Etampes, France) and used as received. Labrafil M1944 CS ${ }^{\circledR}$ (oleoyl macrogolglycerides) and Labrafac $\mathrm{CC}^{\circledR}$ (medium chain triglycerides) were obtained from Gattefossé (Saint-Priest, France). Ketoprofen was kindly gifted by Amoli Organics Ltd. (Mumbai, India). High performance liquid chromatography grade ethanol, vitamin E acetate, sulfamethazine, and tetraglycol were purchased from Sigma (Saint-Louis, USA). Finally, Ultrapure ${ }^{\circledR}$ water was obtained using the MilliQ filtration system (Millipore, Saint-Quentin-en-Yvelines, France).

The different excipients were selected for their compatibility with the aimed administration routes, which are oral and parenteral. The research around these excipients (for instance, nonionic surfactants) was initially developed for human applications, and is potentially compatible with veterinary applications. Thus, the use of tetraglycol in the modified nanoemulsification processes drug solubilization to be enhanced in the nanodroplets (not soluble in pure oils), compatibility with the parenteral administration route to be maintained. Tetraglycol, also called glycofurol or tetrahydrofurfuryl alcohol polyethyleneglycol ether (see Figure 1), is generally regarded as a nontoxic and nonirritant material. It is commonly used as a solvent in parenteral products for intravenous or intramuscular injection ${ }^{3-6}$ in concentrations up to $50 \%(\mathrm{vol} / \mathrm{vol})$. The parenteral administration should not exceed $0.07 \mathrm{~mL} / \mathrm{kg}$ body weight daily.

\section{Low-energy nanoemulsification}

The nanoemulsion droplets are generated according to a simple and efficient method, herein adapted to the encapsulation of 2 drugs of veterinary interest. Low-energy nanoemulsification methods are generally described as follows: 1) spontaneous emulsification and 2) phase-inversion temperature. A detailed description of these formulation processes as well as a comprehensive bibliography can be found in our previous work ${ }^{2}$ and in the works of Salager et $\mathrm{al}^{7}$ and Solans et al. ${ }^{8}$ However, since we have recently shown ${ }^{1}$ that these 2 methods are intimately linked and governed by similar mechanisms, we chose to focus only on spontaneous emulsification, which is the simplest method. The nanoencapsulation processes were tailored to the physicochemical properties of the mol-

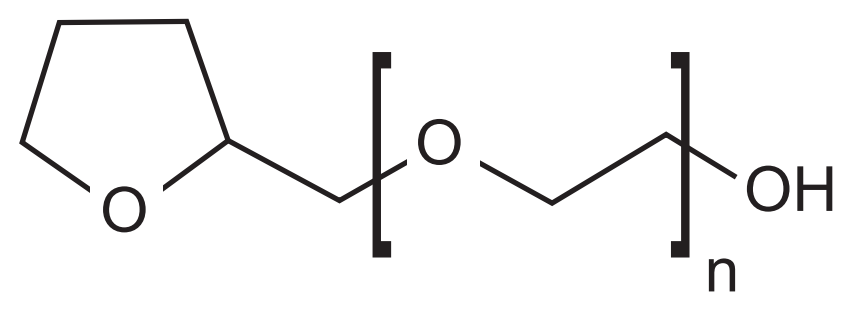

Figure I Structure of tetraglycol, $n=I-2$. 
ecules to be encapsulated within different lipophilic phases (oleoyl macrogolglycerides: Labrafil M $1944 \mathrm{CS}^{\circledR}$, medium chain triglycerides: Labrafac $\mathrm{CC}^{\circledR}$, and vitamin $\mathrm{E}$ acetate), and also with or without the use of an additional substance (ethanol or tetraglycol) for enhancing drug solubilization. As illustrated in Figure 2, the nanoemulsion droplets are generated by bringing two phases into contact: 1) The first phase is composed of oil plus the totally miscible hydrophilic surfactant (plus, optionally, a solvent), solubilizing the active principle ingredient (API);2) The second phase is the aqueous one, which can be, for instance, pure water or buffer.

Once these two phases are mixed, the hydrophilic species are immediately solubilized by the aqueous phase inducing the demixing of the oil (through a spinodal decomposition) in the form of nanometric emulsion droplets. Surfactants immediately stabilize the nanodroplets formed. As presented in Figure 2, the solubilization of APIs (in red) in the organic oily phase (before emulsification) will result in a very homogeneous distribution of APIs in the nanoemulsion droplets.

There are two key conditions in the nanoemulsification process: (1) the drugs' solubilization in the organic phase once this solubilization is achieved achieved, (2) this drug-containing organic phase must still induce the spontaneous emulsifica-

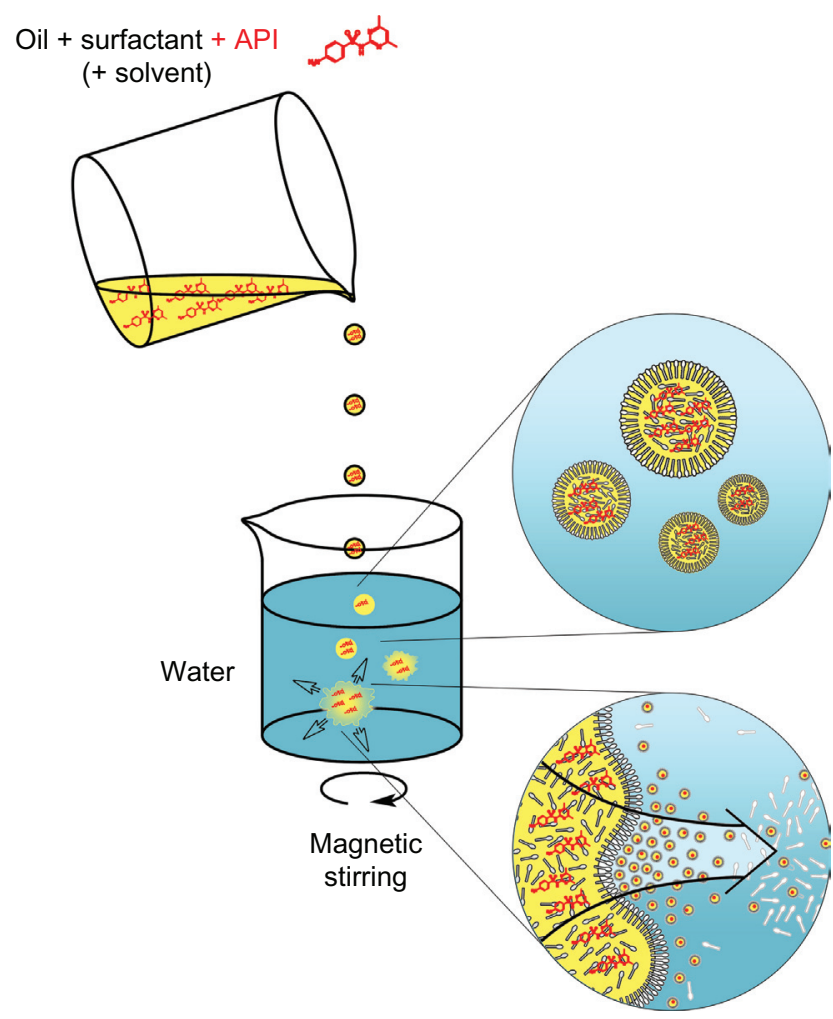

Figure 2 Scheme of the nanoemulsion generating process. Abbreviation: API, active principle ingredient. tion process. These 2 points are thoroughly investigated in the present study. However, the main difficulty arises in the first point because the self-emulsification itself is directly linked to the nature of the oil-nonionic surfactant couple used (and their respective affinities). In this way, adapting the processes in the encapsulation of guest molecules will mainly involve the choice of these latter molecules for the oil-nonionic surfactant couples available. Additional solubilizing substances, which can be a cosolvent, can also be used in the organic phase to enable the drug to be incorporated into oil.

Once the drugs are solubilized in the organic phase (the value of $1 \% \mathrm{wt} / \mathrm{wt}$ in oil is fixed throughout the study), they are homogenized with a vortex mixer and slowly poured into the slightly magnetically stirred $(\sim 300 \mathrm{rpm})$ ultrapure water phase. The nanoemulsion droplets are generated within a few seconds. The samples were stirred for a further 15 minutes.

The different formulations were characterized by determining up the effect of the formulation variables (the relative proportions of the different components) on the nanoemulsions' physicochemical properties (size and polydispersity index [PDI]). We have shown elsewhere ${ }^{1}$ that these 2 points are closely related, and changing the surfactant/oil ratio, for instance, allows for a very precise control of the droplet size and polydispersity. The formulation variables are defined as follows: the solvent/oil weight ratio: $\mathrm{SOR}=100 \times \mathrm{w}_{\text {surfactant }} /\left(\mathrm{w}_{\text {surfactant }}+\mathrm{w}_{\text {oil }}\right)$ and the surfactant-oil/water weight ratio: SOWR $=100 \times \mathrm{w}_{\text {surfactant }+ \text { oil }} /\left(\mathrm{w}_{\text {surfactant }+ \text { oil }}+\mathrm{w}_{\text {water }}\right)$. However, the SOWR has an influence only on the droplet concentration; thus, it was fixed at $30 \%$ throughout this study.

\section{Dynamic light scattering}

Hydrodynamic diameters were obtained by dynamic light scattering using a Malvern NanoZS instrument (Malvern, Orsay, France). The helium-neon laser, $4 \mathrm{~mW}$, operates at $633 \mathrm{~nm}$, with the scatter angle fixed at $173^{\circ}$ and the temperature maintained at $25^{\circ} \mathrm{C}$. PDI is a mathematical definition that accounts for the relative error between curve fit and experimental values. ${ }^{9}$ It shows the quality of the dispersion. Values $\leq 0.15-0.2$ reflect a good monodispersity and quality of the nanoparticulate suspensions. Measurements were performed 3 times for each point.

\section{Results}

\section{Ketoprofen nanoencapsulation}

The first example lies in the encapsulation of a model lipophilic drug, ketoprofen, a nonsteroidal anti-inflammatory drug. The study was first focused on examples of 
oil-nonionic surfactant couples known for being compatible with the spontaneous emulsification process. ${ }^{1}$ These examples are Labrafil M $1944 \mathrm{CS}^{\circledR} / \mathrm{Cremophor}^{\mathrm{ELP}^{\circledR}}{ }^{\circledR}$ and Labrafac $\mathrm{CC}^{\circledR} /$ Solutol HS15 ${ }^{\circledR}$. It is noteworthy that many possibilities exist between these families of excipients, and the examples studied herein are neither exhaustive nor representative. Point 1) mentioned regarding the drug solubilization in the oily phase is rapidly achieved, showing the compatibility of ketoprofen with these kinds of hydrophilic phases. For point 2), however, the influence of drug solubilization on the emulsification process itself was investigated by comparing nanoemulsions formed without ${ }^{1}$ and with the presence of drugs. The results are reported in Figures 3A and $3 \mathrm{~B}$, showing the droplet hydrodynamic diameter plotted against the surfactant/oil ratio denoted SOR (see Materials and Methods section).

The curves describe a continuous behavior from micrometric droplets continuously and rapidly reaching the nanoscale as the SOR is increased, along with a good monodispersity of the samples. Also, droplet sizes and PDI can be controlled very easily by changing only one formulation parameter, SOR. Another important result is the close similarity of the physicochemical properties of the drug-loaded nanoemulsions or nonloaded nanoemulsions, and regardless of the system. Furthermore, the quality of the emulsification, shown through the PDI, is conserved between loaded and nonloaded nanoemulsions.

\section{Sulfamethazine nanoencapsulation}

The second example of drug nanoencapsulation uses sulfamethazine, which is a veterinary antibacterial drug used in breeding animals. This molecule was selected to illustrate the experimental case for which drug solubilization is not simply achieved in the pure oily phase but needs the help of an additional cosolvent. Therefore, two cosolvents were tested: one is ethanol used with the combination Labrafil M 1944 CS $^{\circledR} /$ Cremophor ELP ${ }^{\circledR}$ (Figure 4); the other is tetraglycol, a solvent also compatible with parenteral administration (described in the Meterials and Methods section), used with two examples of oil-surfactants combinations: Labrafac $\mathrm{CC}^{\circledR} /$ Cremophor $\mathrm{ELP}^{\circledR}$ (Figure $5 \mathrm{~A}$ ), and vitamin E acetate/Cremophor ELP ${ }^{\circledR}$ (Figure 5B).

The solubilization of sulfamethazine in the selected representative oil phases, which relatively differs according to the nature of the cosolvent. Ethanol and tetraglycol have been shown to fulfill this objective in all the phases tested, which are Labrafil M $1944 \mathrm{CS}^{\circledR}$, Labrafac $\mathrm{CC}^{\circledR}$, and vitamin E acetate.
Similar to the results obtained with ketoprofen, the curves show a constant gradual decrease of the nanoemulsion droplet size as the surfactant amount increases. Furthermore, the gray parts show at what point the nanodroplets form and

\section{A)}

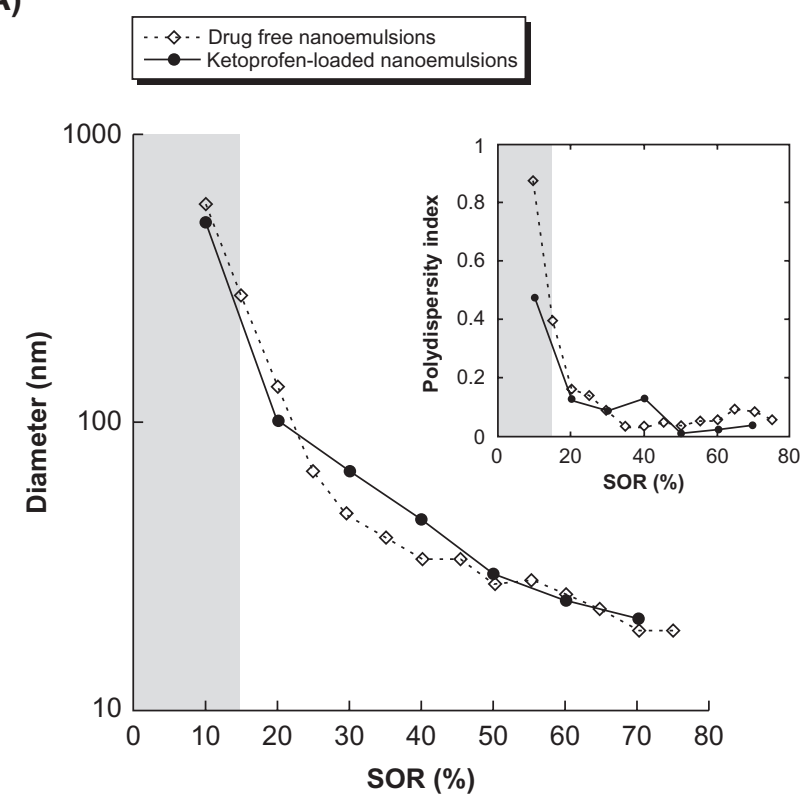

B)

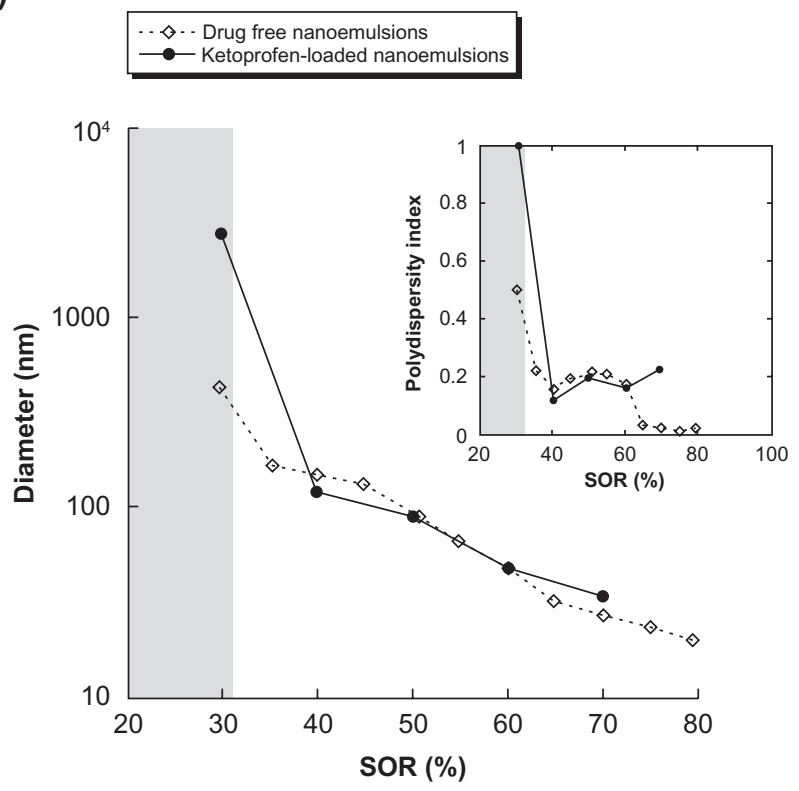

Figure 3 Nanoemulsions formulated with low-energy spontaneous emulsification. A) Surfactant $=$ Cremophor ELP ${ }^{\circledast}$, oil $=$ Labrafil M I 944 CS $^{\circledR}$. B) Surfactant $=$ Solutol $\mathrm{HSI}$, oil = Labrafac CC ${ }^{\circledR}$. Hydrodynamic diameter and polydispersity index (PDI; inset) are plotted against the surfactant/oil weight ratio (SOR). Nanoemulsionsencapsulating the drug (diclofenac) are shown with the filled circles and compared with a formulation of "empty" nanoemulsions (open diamond-shaped symbols). The gray parts indicate that the criteria of PDI quality are not met, and the suspension cannot be considered as a nanoemulsion. Relative standard deviations are comprised as follows: A) for empty nanoemulsions, between $1.5 \%$ and $0.2 \%$; and for loaded nanoemulsions, between $4.3 \%$ and $3.5 \%$; B) for empty nanoemulsions, between $1.3 \%$ and $0.1 \%$; and for loaded nanoemulsions, between $3.8 \%$ and $2.2 \%$. 


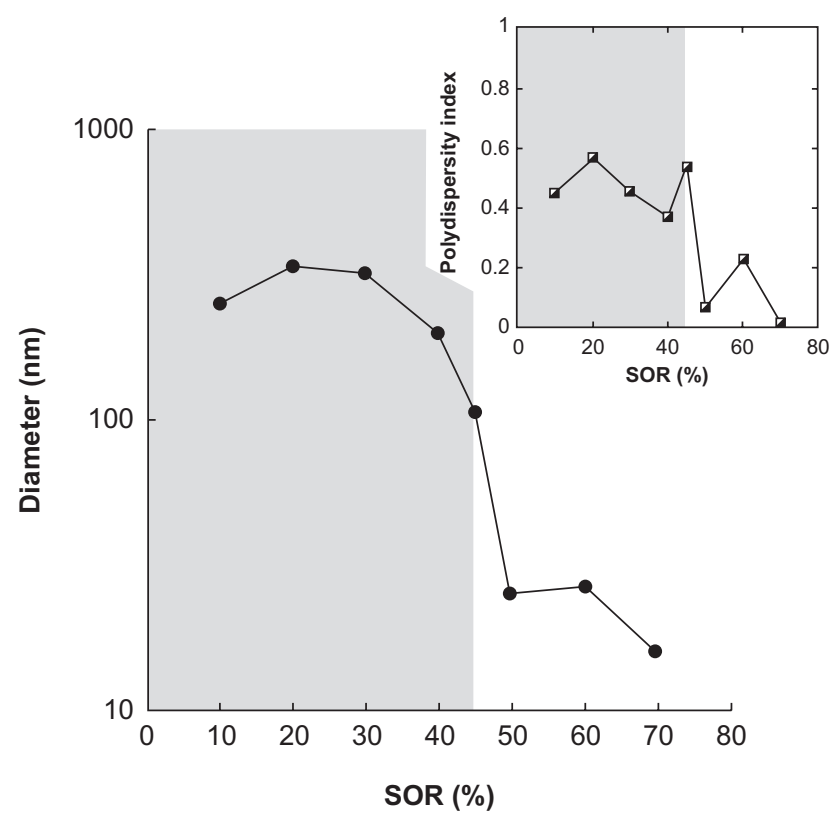

Figure 4 Encapsulation of sulfamethazine in nanoemulsions formulated with lowenergy spontaneous emulsification, with ethanol as a cosolvent. Hydrodynamic diameter and polydispersity index (PDI; inset) are plotted against the surfactant oil weight ratio (SOR). Surfactant $=$ Cremophor ELP ${ }^{\circledR}$, oil $=$ Labrafil M 1944 CS $^{\circledR}$. Oil/cosolvent weight ratio $=1$. The gray part indicates that the criteria of PDI quality are not met, and the suspension cannot be considered as a nanoemulsion.

meet the quality criteria (low PDI). The results in Figures 4 and 5 show that this limit can be strongly affected by the nature of the oil-surfactant combinations: a SOR of around $45 \%$ in Figure 4, and around $40 \%$ and $20 \%$ in Figures $5 \mathrm{~A}$ and $5 \mathrm{~B}$, respectively.

\section{Discussion}

Low-energy nanoemulsification is a powerful process for generating stable nanodispersion of lipophilic compounds in an aqueous media, and at low energy costs. In addition, the strong interest in nanoemulsions for such applications also comes from their high degree of stability in suspension, maintained months. This stability is the consequence of the extremely small size of the nanoemulsion droplets which render the Brownian particles insensitive to the gravitational effect and thus to the subsequent destabilization processes such as flocculation (and coalescence) of concentrated droplets. Only Ostwald ripening (the diffusion of the dispersed phase through the bulk phase) acts to destabilize the nanoemulsion droplets, ${ }^{2}$ which is very low, and therefore very relevant to our intended applications. The numerous advantages of low-energy nanoemulsification appear when the process is compared with the commonly found processes used to generate nanoparticulate drug delivery systems, which are generally high-energy lipid emulsification most
A)

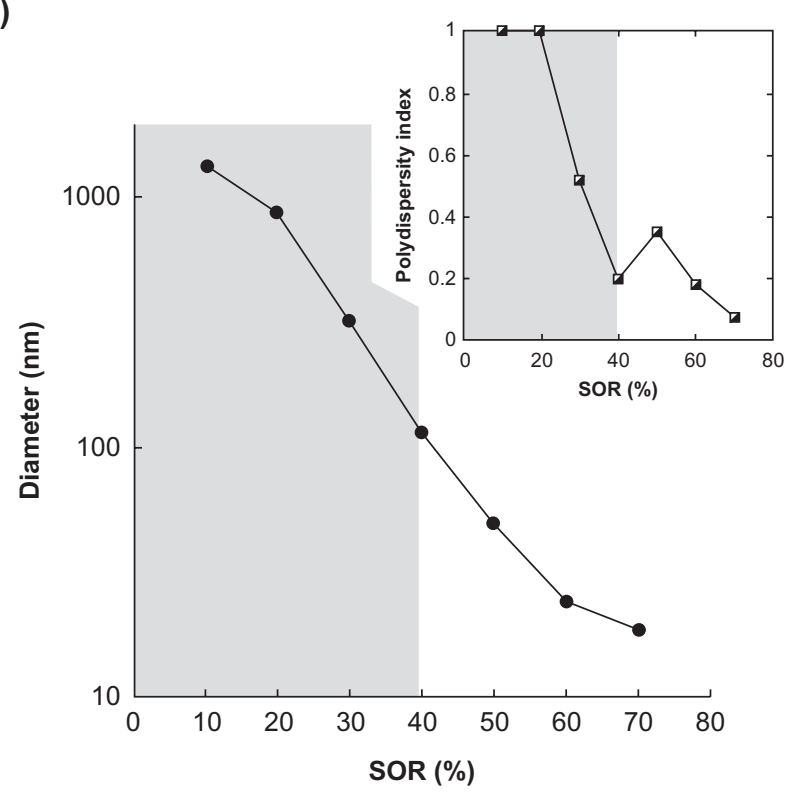

B)

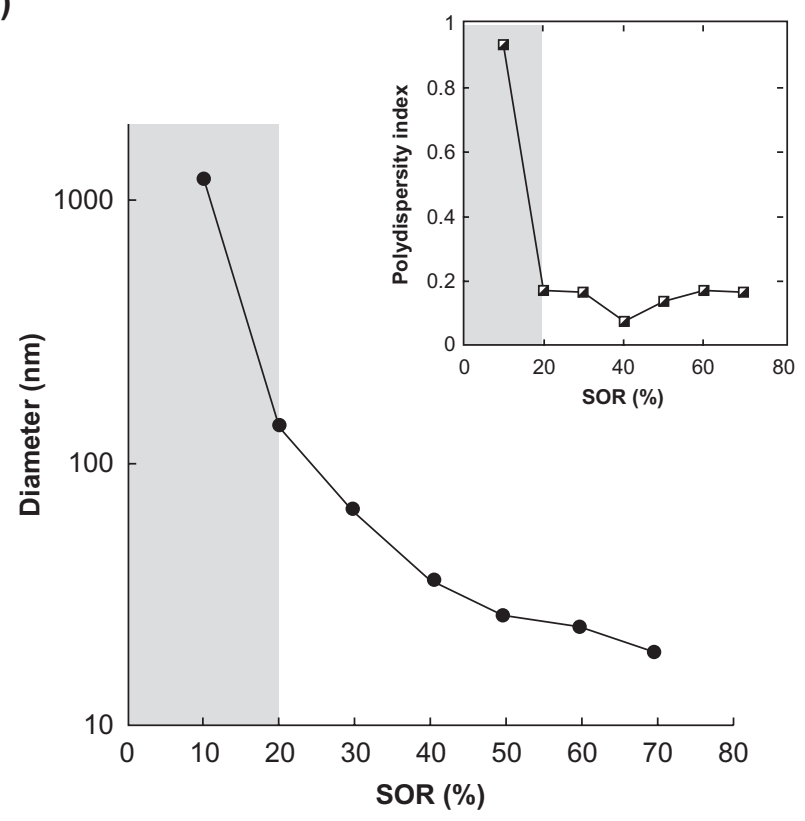

Figure 5 Encapsulation of sulfamethazine in nanoemulsions formulated with lowenergy spontaneous emulsification, with tetraglycol as a cosolvent. Hydrodynamic diameter and polydispersity index (PDI; inset) are plotted against the surfactant/ oil weight ratio (SOR). A) Surfactant $=$ Cremophor ELP ${ }^{\circledR}$, oil $=$ Labrafac CC $^{\circledR}$. B) Surfactant $=$ Cremophor ELP ${ }^{\circledR}$, oil $=$ vitamin E acetate. Oil/cosolvent weight ratio $=\mathrm{I}$. The gray parts indicate that the criteria of PDI quality are not met, and the suspension cannot be considered as a nanoemulsion.

often polymeric, ${ }^{2}$ or even inorganic chemistry through, for instance, a sol-gel process described as a 2-step polycondensation forming silica particles. ${ }^{10}$ Low-energy nanoemulsification does not need any specific devices or chemical reactions for the formation of the colloidal objects: the simplicity of the whole process as well as a high energy yield, constitute significant advantages of this technology. 
However, the encapsulation of a given lipophilic active molecule into nanoemulsion droplets for their homogeneous dispersion in water has been shown to strongly depend on the physicochemical properties (and thus on the nature) of the excipients used. Indeed, it depends on the solubilization of such active molecules in the oil used in the nanoemulsion formulation, potentially with the help of a cosolvent. Accordingly, the scientific approach chosen consists of adapting the low-energy nanoemulsification process to the molecule to be encapsulated, involving the use of a cosolvent for enhancing drug solubilization.

The formulation of lipophilic molecules is potentially dedicated to both parenteral and oral administration routes: the operator himself performs the animal injection in the first case, or uses a method of dispersion into the beverage of breeding animals. However, a important problem linked to the formulation of the lipophilic compounds persists with conventional technologies, since it is estimated that $>40 \%$ of new pharmacologically active chemical entities identified by high-throughput screening have a major problem with their water solubility. ${ }^{11}$ In this context, low-energy nanoemulsification technology could offer interesting solutions to this problem.

The two examples of molecules presented in the present study, ketoprofen and sulfamethazine, provide representative cases of such formulations of lipophilic compounds. Thus, they can show how the methodology for adapting the emulsification processes to the bioactive molecule properties can be applied, and illustrate how these nanoemulsification can be characterized globally (through the effect of the SOR on the formulation). It appears (Figure 3) that drug encapsulation does not influence the global nanoemulsion formulation in the absence of additional cosolvent, showing the adaptability of the method when the drug is easily soluble in the oily phase. However, the presence of a cosolvent in the formulation appears to strongly influence the process as illustrated by the difference between Figure 3A (solvent-free formulation) and Figure 4 (with ethanol as a solubilizing enhancer for sulfamethazine) for the same oil/surfactant combination (Labrafil M $1944 \mathrm{CS}^{\circledR} /$ Cremophor ELP ${ }^{\circledR}$ ).

The limit in SOR for which the emulsion droplets become submicronic is shifted from $15 \%$ to $45 \%$. In addition, the low PDIs observed, even when using a cosolvent (meaning when the APIs are poorly soluble in the oil), testify that the drug has not recrystallized in an anarchical way, but rather it is well encapsulated. This results in an increase in the amount of the surfactant in the formulations and has to be taken into account in the evaluation of the excipient dosage per animal. For the example oral formulations ie, nanoemulsions added to the beverages of breeding animals, the specifications only require the droplets to have a size below the micrometer dimension with high monodispersity, in order to ensure a high suspension stability. All the results (Figures 3-5) show that, this is obtained at an SOR shown by the limit between the gray and white areas: actually this limit could constitute the optimized formulation since it is a compromise that meets the specifications in terms of size and polydispersity, with the lowest amount of surfactant.

However, this so-called low-energy method means that the energy yields are extremely favorable for an industrial scale-up, since the emulsification does not need any mechanical mixing. This point is all the more important for veterinary compared with human applications since the orders of magnitude of the number of simultaneous administrations are significantly different. The 2 phases (organic and aqueous), once mixed, have to be homogenized, and this stage of the formulation is the only stage that needs a given (but low) amount of energy. A simple schematic solution is proposed in Figure 6.

Figure 6 shows a simple and schematic solution for the automated in situ emulsification and delivery of drugs in nanoemulsions added to beverages of breeding animals (domestic pigeons). The two immiscible phases are brought into contact through a system of 2 pipes, at constant flux. The proper functioning of the system is ensured by the 2 nonreturn valves before the emulsifying chamber. Once the emulsification is initiated, the homogenization is performed in the next homogenization chamber with liquid turbulences, which are induced by successive changes in the liquid velocity, created by successive bottlenecks in the pipe. The homogeneous dispersion of drug-loaded nanoemulsions is then transported toward the drinking trough.

To conclude, such a technology could also be used to administer one or several drugs simultaneously either by adding a pipe or by adding a transport system. As well, one can imagine that one formulation developed for multiple applications, which either can be used for different animal species or can be adapted to animals of different weights (by changing the automated dosage instructions).

\section{Conclusion}

In this study, we present results that demonstrate the potential of low-energy nanoemulsification for veterinary applications. We present an overview of the feasibility and limits of the encapsulation of bioactive molecules through this technology. 


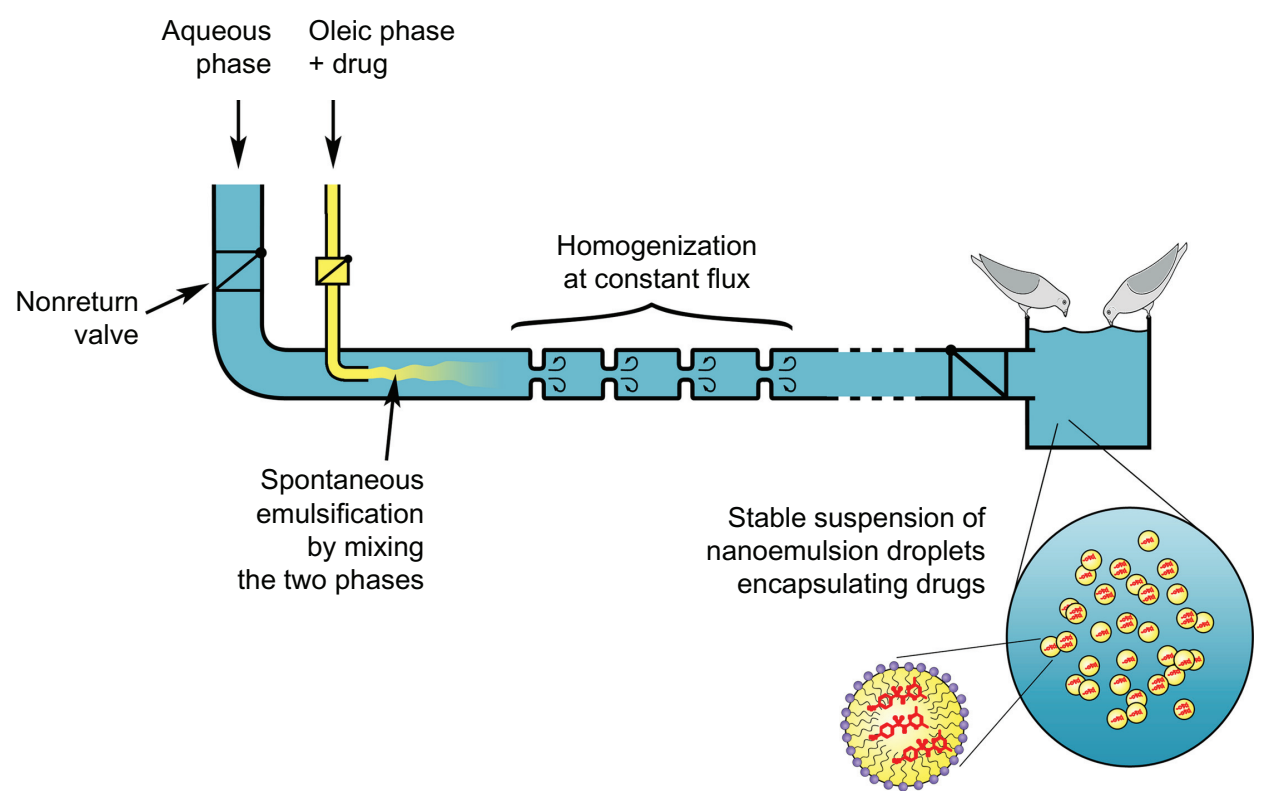

Figure 6 Schematic design of automated in situ emulsification and delivery of drugs (red) in nanoemulsions (yellow) into beverages of breeding animals.

The general technique consists of adapting the formulation process to the molecule to be encapsulated, which can be achieved with or without the help of an additional cosolvent (ethanol), as well as an compatible substance that plays the same role (tetraglycol). Finally, this study shows the many advantages of the low-energy nanoemulsification method, specifically for veterinary applications generally involved in large-scale drug delivery.

\section{Disclosure}

The authors report no conflicts of interest in this work.

\section{References}

1. Anton N, Vandamme TF. The universality of low-energy nanoemulsification. Int J Pharm. 2009;377:142-147.

2. Anton N, Benoit JP, Saulnier P. Design and production of nanoparticles formulated from nano-emulsion templates-a review. $J$ Control Release. 2008;128:185-199.

3. Weller PJ, Glycofurol. In: Rowe RC, Sheskey PJ, Owen SC, editors. Handbook of Pharmacentical Excipients. 5th ed. 2006:313-314.

4. Spiegelberg H, Schläpfer R, Zbinden G, Studer A. A new injectable solvent (glycofurol) [in German]. Arzneimittelforschung. 1956;6:75-77.
5. Spiegel AJ, Noseworthy MM. Use of non-aqueous solvents in parenteral products. J Pharm Sci. 1963;52:917-927.

6. Dale O, Sheffels P, Khorasch ED. Bioavailabilities of rectal and oral methadone in healthy subjects. Br J Clin Pharmacol. 2004;58(2):156-162.

7. Salager JL, Forgiarini A, Marquez L, et al. Using emulsion inversion in industrial processes. Adv Colloid Interface Sci. 2004;108-109: 259-272.

8. Solans C, Izquierdo P, Nolla J, Azemar N, Garcia-Celma M-J. Nano-emulsions. Curr Opin Colloid Interface Sci. 2005;10:102-110.

9. Anton N, Gayet P, Benoit JP, Saulnier P. Nano-emulsions and nanocapsules by the PIT method: an investigation on the role of the temperature cycling on the emulsion phase inversion. Int J Pharm. 2007;344:44-52.

10. Barbé C, Bartlett J, Kong L, et al. Sillica particles: a novel drug delivery system. Adv Mater. 2004;16:1963-1966.

11. Lipinski CA, Lombardo F, Dominy BW, Feeney PJ. Experimental and computational approaches to estimate solubility and permeability in drug discovery and development settings. Adv Drug Deliv Rev. 2001; 46(1-3):3-26.
International Journal of Nanomedicine

\section{Publish your work in this journal}

The International Journal of Nanomedicine is an international, peerreviewed journal focusing on the application of nanotechnology in diagnostics, therapeutics, and drug delivery systems throughout the biomedical field. This journal is indexed on PubMed Central, MedLine, CAS, SciSearch ${ }^{\circledR}$, Current Contents ${ }^{\circledR} /$ Clinical Medicine,

\section{Dovepress}

Journal Citation Reports/Science Edition, EMBase, Scopus and the Elsevier Bibliographic databases. The manuscript management system is completely online and includes a very quick and fair peer-review system, which is all easy to use. Visit http://www.dovepress.com/ testimonials.php to read real quotes from published authors. 